\title{
Adsorption of Natural Organic Matter onto Carbonaceous Surfaces: An Atomic Force Microscopy Study
}

\author{
Justin M. Gorham, Joshua D. Wnuk, M. Shin, \\ Howard Fairbrother* \\ Department of Chemistry \\ Johns Hopkins University, 3400 N. Charles St., Baltimore, MD 21218 \\ *Corresponding author email address: howardf@jhu.edu \\ Phone: (410) 516-4328; fax: (410) 516-8420 \\ Submitted to Environmental Science and Technology \\ July, 2006
}

Revised, October 24, 2006

\section{Supporting Information:}

\author{
5 Pages \\ 4 Figures \\ 1 Table
}


Table S-1: Composition of Dismal Swamp Water (DSW)

\begin{tabular}{|l|c|c|}
\hline \multicolumn{1}{|c|}{ Component } & Concentration & Units \\
\hline Organic materials: & & \\
Dissolved Organic Carbon (DOC) & $98 \sim 100$ & $\mathrm{mg} \mathrm{C/L}$ \\
UV $_{\text {254 }}$ & 4.52 & $\mathrm{~cm}^{-1}$ \\
Hydrophobic/hydrophilic fraction & $64 \% / 36 \%$ & \\
Charge density & -15 at pH 10 & $\mathrm{meq} / \mathrm{gC}$ \\
& -11 at pH 7 & $\mathrm{meq} / \mathrm{gC}$ \\
& -5 at $\mathrm{pH} \mathrm{4}$ & $\mathrm{meq} / \mathrm{gC}$ \\
\hline Inorganic ions: & & \\
Ca(II) & $0.12 \sim 0.16$ & $\mathrm{mM}$ \\
Mg(II) & 0.10 & $\mathrm{mM}$ \\
Fe(III) & 0.048 & $\mathrm{mM}$ \\
Al(III) & 0.032 & $\mathrm{mM}$ \\
$\mathrm{Na}^{+}$ & 0.24 & $\mathrm{mM}$ \\
$\mathrm{Cl}^{-}$ & 0.27 & $\mathrm{mM}$ \\
\hline
\end{tabular}

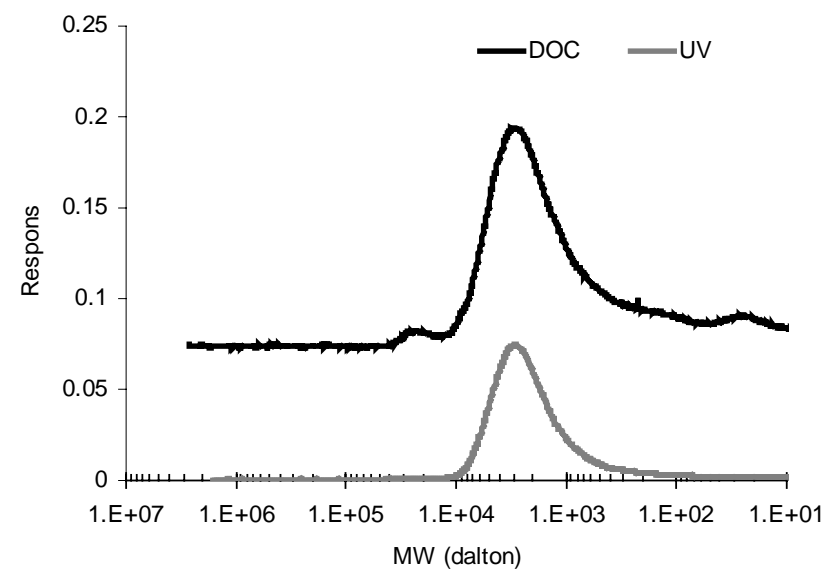

Figure S-1: Size Exclusion Chromatograph of Dismal Swamp Water. 


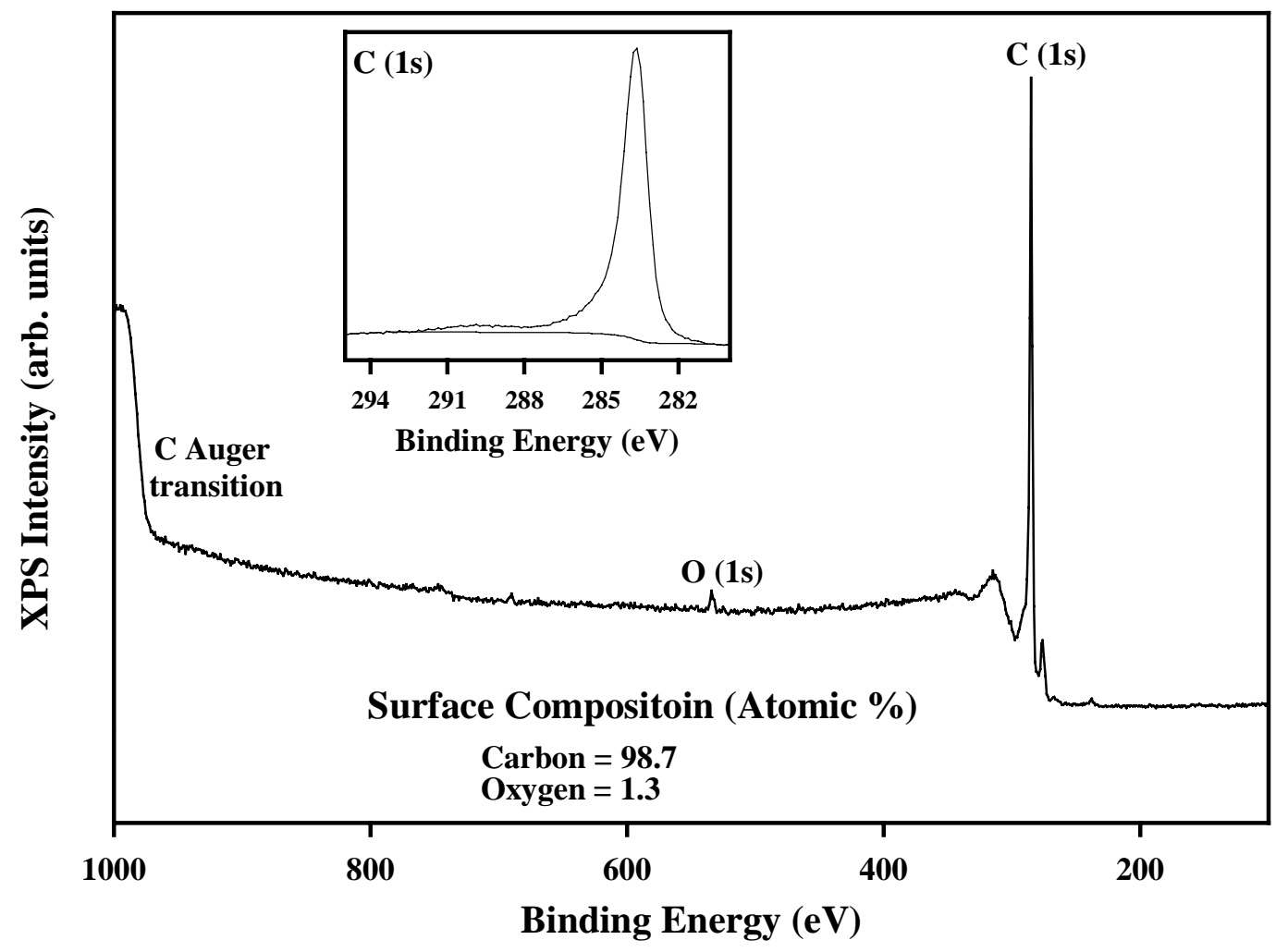

Figure S-2: X-ray Photoelectron Spectra (100-1000eV binding energy) of HOPG (highly ordered pyrolitic graphite) showing the chemically well-defined nature of the substrate and the lack of any surface oxides. A more detailed analysis of the $\mathrm{C}(1 \mathrm{~s})$ XPS spectral region is also shown. 

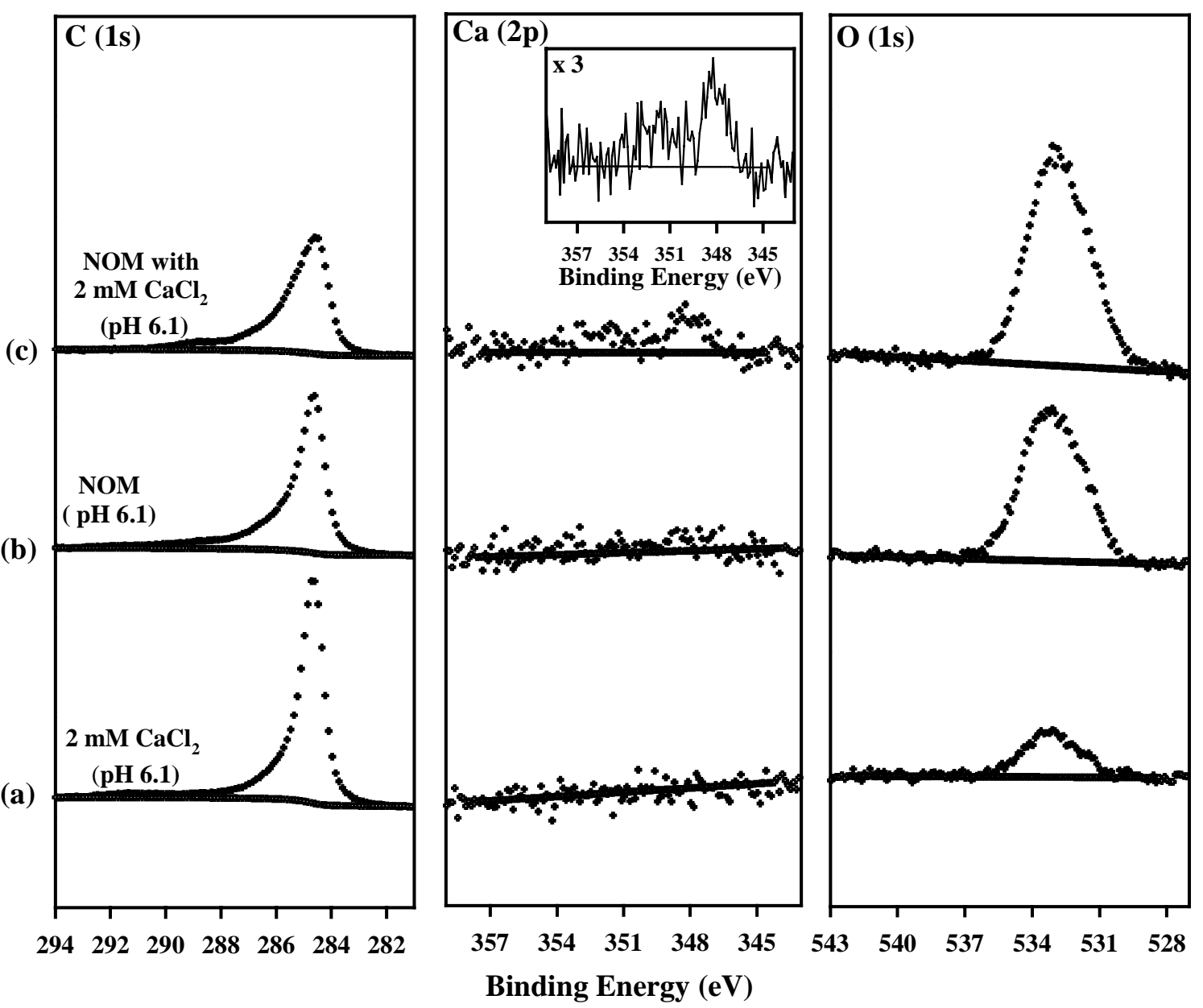

Figure S-3: Change in the surface composition of HOPG following exposure to different aqueous conditions, (a) $2 \mathrm{mM} \mathrm{CaCl}_{2}$, (b) NOM within the GDS and (c) $2 \mathrm{mM} \mathrm{CaCl}_{2}$ with NOM. In each experiment a freshly prepared HOPG substrate was immersed in solution for one hour. The sample was then removed from solution, washed with copious quantities of milli-Q water before the surface was analyzed under vacuum with XPS. 
(a)
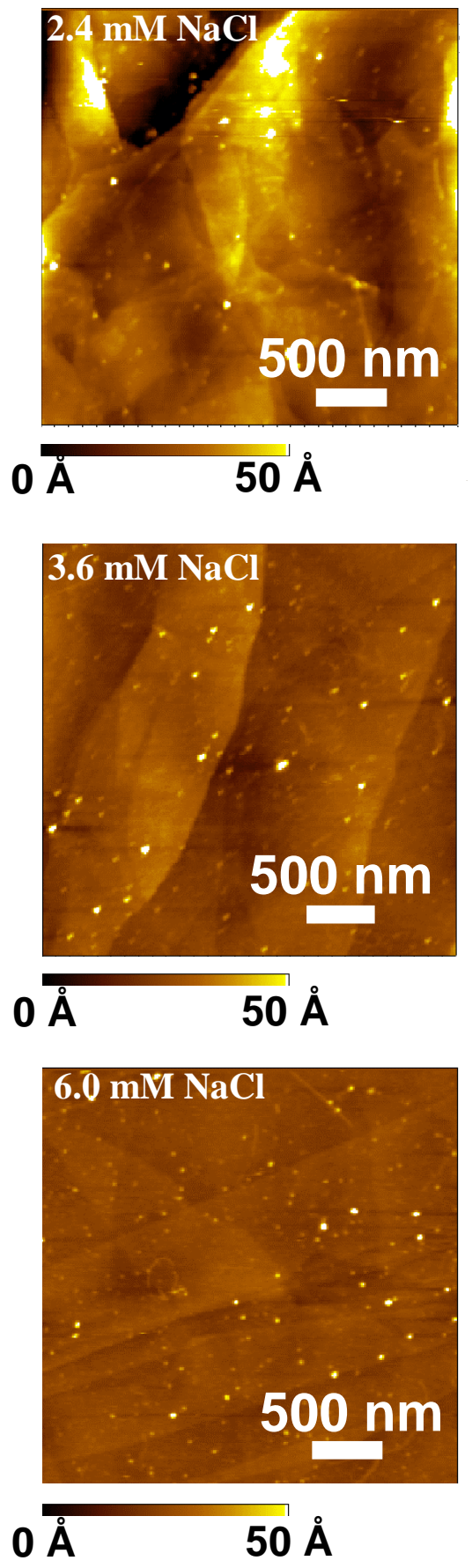

(b)
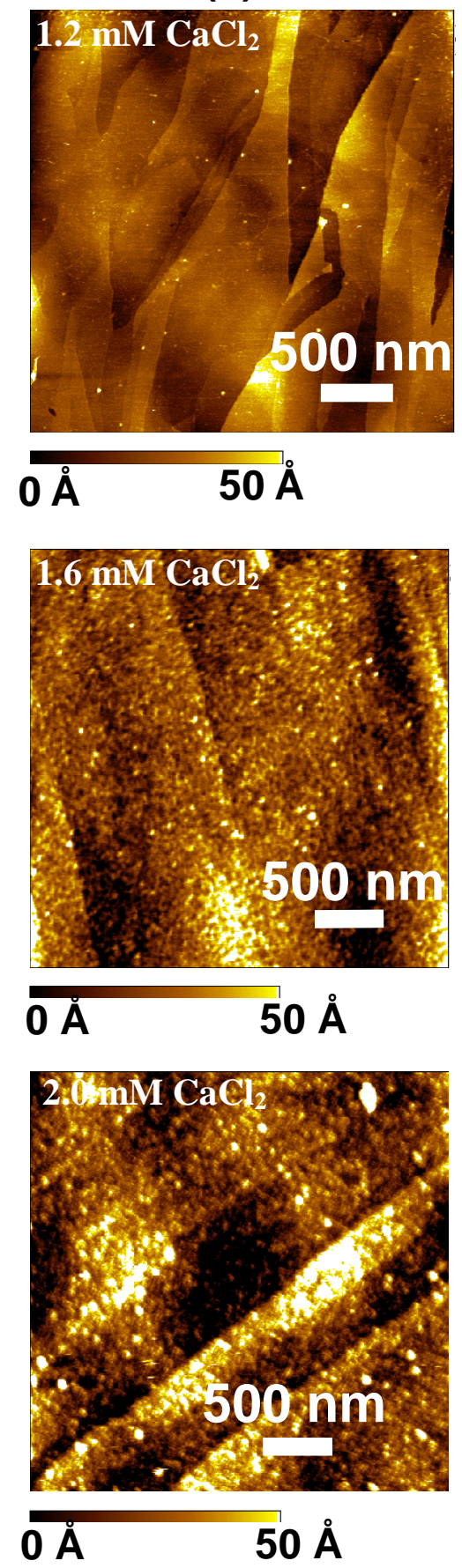

Figure S-4: Topographic AFM images showing the influence of (a) ionic strength and (b) $\mathrm{Ca}^{2+}$ concentration on the adsorption of NOM onto HOPG. In each experiment the $\mathrm{pH}$ was 6.3 . 